Radiologe 2012 $\cdot 52: 615-620$

DOI 10.1007/s00117-011-2272-1

Online publiziert: 18 . Juli 2012

(c) Springer-Verlag 2012

B. Klaeser · M. Spanjol · T. Krause

Universitätsklinik für Nuklearmedizin, Inselspital Bern, Bern

\title{
SPECT/CT-Infektdiagnostik am Skelett
}

lettszintigraphie bei orthopädischen und onkologischen Fragestellungen. Die $\mathrm{Zu}$ satzinformationen der SPECT/CT lassen hier zuvor unklare Befunde meist eindeutig zuordnen und modifizieren die Diagnose auf der Basis der planaren Aufnahmen in rund einem Drittel der Fälle [11, 17].

Die Anzahl wissenschaftlicher Arbeiten zum Stellenwert der SPECT/CT für die Infektbildgebung ist gegenwärtig noch begrenzt $[1,4,5,6,7,8,9]$. Die vorliegende Arbeit gibt aus diesem Grund neben Erkenntnissen der publizierten Literatur auch persönliche Erfahrungen und Einschätzungen der Autoren wieder.

\section{Methodik der nuklearmedizinischen Infektbildgebung}

Die frühe und korrekte Diagnose und Behandlung von Knocheninfekten ist von großer Bedeutung und hat entscheidenden Einfluss auf Therapiewahl und Komplikationsrate. Während die Diagnose akuter und postoperativer Gelenk- und Knocheninfekte in der Regel problemlos gestellt werden kann, bereitet die Diagnose chronischer Knocheninfekte nicht selten Schwierigkeiten. Die Diagnose chronischer Infekte beruht neben Laborparametern und Bakteriologie v. a. auf den Befunden radiologischer und nuklearmedizinischer Verfahren.

Die nuklearmedizinische Bildgebung hat sich mit der Einführung von Hybridtechnologien wie der „single photon emission computed tomography“/CT (SPECT/ CT) tiefgreifend verändert. Die SPECT/ CT steigert allgemein die diagnostische Genauigkeit nuklearmedizinischer Verfahren erheblich, z. B. diejenige der Ske-
Die konventionelle nuklearmedizinische Infektbildgebung umfasst mehrere Untersuchungstypen mit verschiedenen Radiopharmazeutika, z. B. ${ }^{67} \mathrm{Ga}$-Zitrat, osteotropen Phosphonatverbindungen, in-vitro-markierten autologen Leukozyten, monoklonalen Antigranulozytenantikörpern und radiomarkierten Antibiotika. Diese Untersuchungen unterscheiden sich hinsichtlich ihres Untersuchungsprinzips und weisen $u$. a. die Mehrdurchblutung in entzündetem Gewebe, die osteoplastische Reaktion bei Knocheninfekten und die Ansammlung von Entzündungszellen im Infekt nach. In der Routinediagnostik kommen v. a. die Mehrphasenskelettszintigraphie (MPSS) mit Phosphonatverbindungen und die Infektszintigraphie (IS) mit radiomarkierten Leukozyten bzw. Antigranulozytenantikörpern zur Anwendung.

\section{Mehrphasenskelettszintigraphie (MPSS)}

Bei der MPSS werden nach Injektion des osteotropen Radiopharmakons dynamische Aufnahmen während der arteriellen Phase (1. Minute p.i., Radionuklidangiographie bzw. Perfusionsphase) und statische Aufnahmen während der venösen Phase (2. bis ca. 6. Minute p.i., Weichteilphase, gesteigerte Blutfülle und/oder Radiopharmakonexsudation) akquiriert. Die Spätaufnahmen während der ossären bzw. Mineralisationsphase zeigen die regionale Knochenstoffwechselaktivität des Skeletts (3-5 h p.i.). Die Basis der MPSS bilden planare Aufnahmen, die bei hoher räumlicher Auflösung zeiteffiziente Übersichtsaufnahmen erlauben. Diese werden bei Bedarf durch Schichtaufnahmen mittels SPECT ergänzt. Eine negative MPSS schließt einen knöchernen Infekt mit hoher Wahrscheinlichkeit aus (negativer prädiktiver Vorhersagewert [NPV] 96\% [16]), sodass sich die MPSS sehr gut als Eingangsuntersuchung zum Infektausschluss und zur Lenkung weiterer Abklärungen eignet. Die Spezifität der MPSS ist dabei direkt an die individuelle Fragestellung und Vorgeschichte gekoppelt, da die beurteilten Parameter unter methodischen Gesichtspunkten nicht infektspezifisch sind. So findet sich eine gesteigerte Gewebedurchblutung z. B. auch im Rahmen reaktiver entzündlicher Prozesse.

\section{Infektszintigraphie (IS)}

Knochenstoffwechselsteigerungen in der Skelettszintigraphie können aus verschiedenen Ursachen vorbestehen und nach einem Ereignis über Monate fortbeste- 

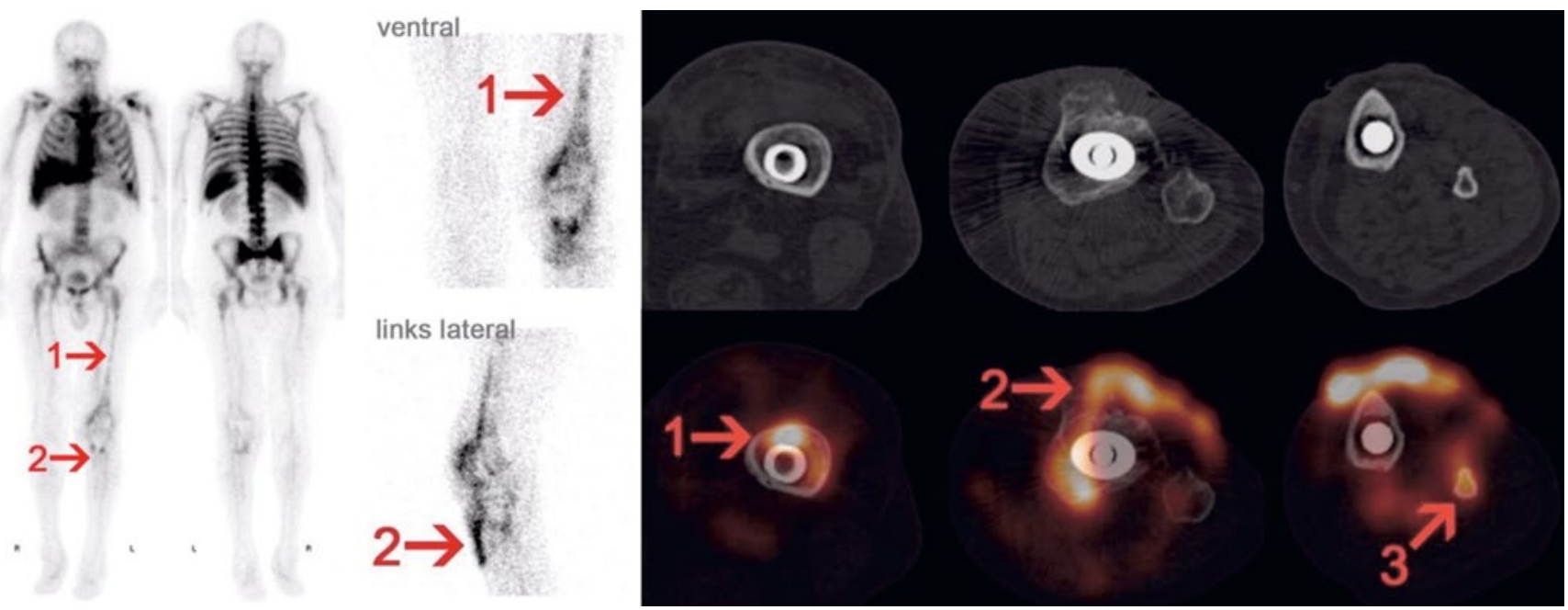

Abb. $1 \Delta$ a, b Chronischer Infekt der Knie-TEP links seit 2 Jahren. Hautfistel an der Tibiavorderseite. In der planaren IS inhomogene Anreicherung in Projektion auf den Femurschaft (1), infektbedingt oder veränderte Knochenmarkverteilung nach mehreren Eingriffen. Ferner infektverdächtige Anreicherung periprothetisch in Projektion auf die proximale Tibia (2). In der ISSPECT/CT infekttypische Mehranreicherung periprothetisch kortikal im Femurschaft (1), in der proximalen der Tibia mit Fistelgang zur Haut ventral (2) sowie Infektausbreitung interfaszial im Bereich der Wade unter Einbezug der Fibula (3). TEPTotalendoprothese, IS Infektszintigraphie, SPECT/CT "single photon emission computed tomography"/CT
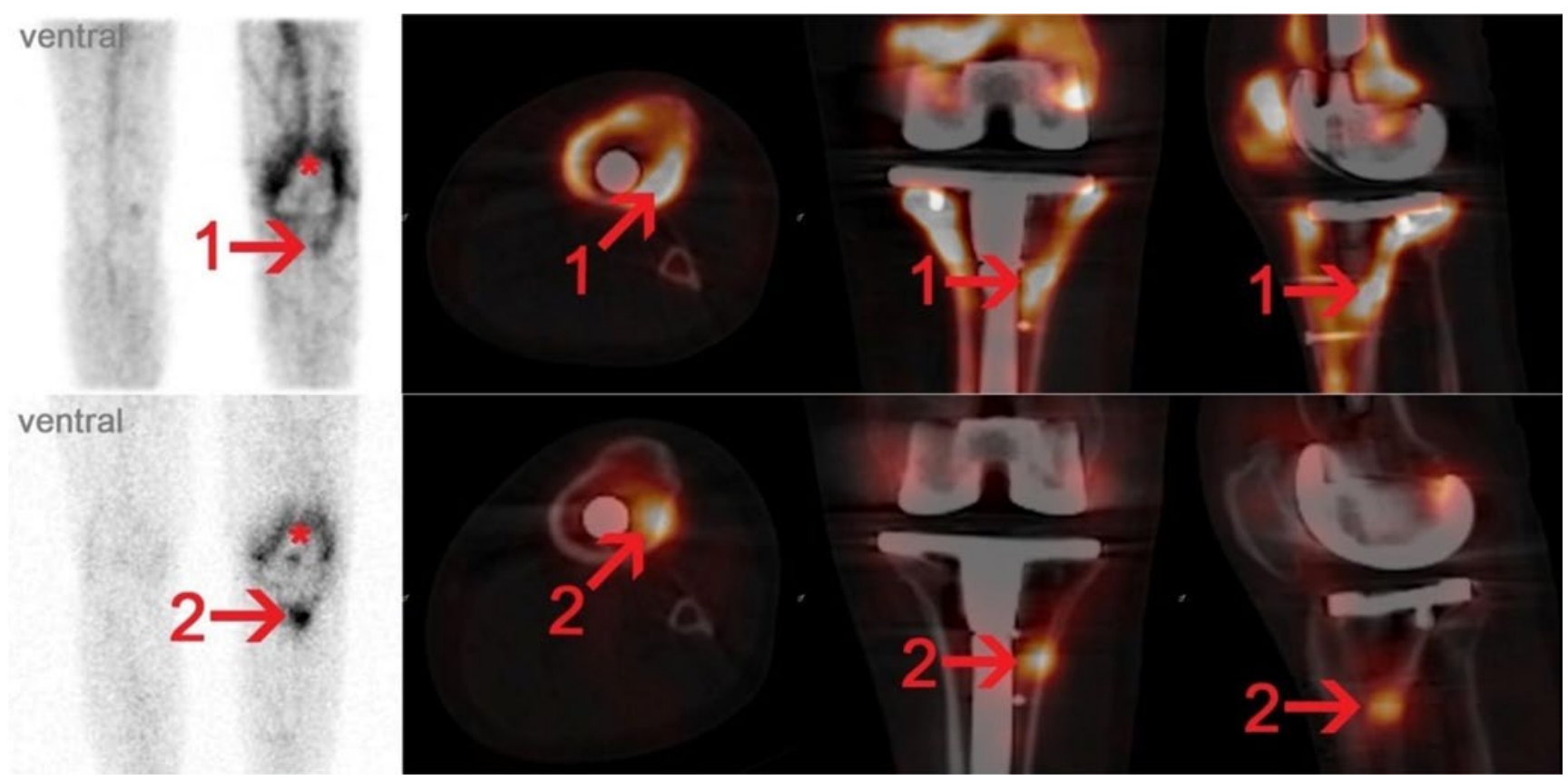

Abb. $2 \Delta$ Zustand nach Knie-TEP-Implantation und mehreren Revisionen. Frage nach Infekt. In der MPSS während der venösen Phase (obere Reihe) neben einer ausgeprägten Synovialitis $\left(^{*}\right)$ fokal gesteigerte Durchblutung ossär angrenzend an den tibialen Prothesenschaft (1). In der IS (untere Reihe) Bestätigung eines Protheseninfekts in dieser Lokalisation (2). Unspezifische synoviale Anreicherung geringerer Intensität bei reaktiver Synovialitis (*). TEP Totalendoprothese, MPSS Mehrphasenskelettszintigraphie, IS Infektszintigraphie

hen. Bei unverletztem und nicht voroperiertem Skelett liegt die Spezifität der planaren MPSS bei $92-95 \%[12,13,19]$, bei Zustand nach Trauma, Operation oder bei chronischem Infekt jedoch je nach Situation unter 50\% [19, 21]. In solchen Fällen erlaubt die IS, die Ansammlungen markierter Leukozyten im Infekt nachweist, eine spezifischere Beurteilung. Kommerziell erhältliche Kitpräparationen für die In-vivo-Markierung von Granulozyten bieten eine ähnliche diagnostische Genauigkeit wie die aufwendigere In-vitro-Markierung autologer Leukozyten [2, $3,15]$ und ermöglichen Infektszintigraphien auch in Instituten ohne entspre- chende Laborausstattung. Die Aufnahmezeitpunkte bei der im deutschsprachigen Raum hauptsächlich verwendeten IS mit Antigranulozytenantikörpern sind bei 4-6 und $24 \mathrm{~h}$ p.i. Die Sensitivität und die Spezifität der IS in planarer Technik liegen im Durchschnitt bei 81-83 bzw. 77-82\% $[14,18,20]$, jedoch mit erheblicher Varia- 
tion abhängig von der Lokalisation des Infekts. So beträgt die Sensitivität der IS bei einer Pathologie im Bereich der Extremitäten durchschnittlich $87 \%$, in der Wirbelsäule dagegen lediglich 53\%. Ursache ist u. a. die Markierung von Granulozyten und Vorläuferzellen im normalen Knochenmark. Knochenmarkanreicherungen können umgekehrt als Infekt fehlgedeutet werden und bedingen eine niedrigere Spezifität der IS in Torso und Wirbelsäule. Infolge veränderter Knochenmarkverteilung nach Trauma oder Prothesenimplantation kann ebenso die Zuordnung atypischer Knochenmarkanreicherungen in den Extremitäten Schwierigkeiten bereiten.

Zusätzliche SPECT-Aufnahmen sind nützlich, um die Lokalisation von Anreicherungen in der IS genauer zu bestimmen, z. B. bei Überlagerungen des Stammskeletts in den planaren Projektionsaufnahmen und zur Unterscheidung von Knochen- gegenüber Weichteilanreicherungen. Die SPECT allein ist jedoch nicht geeignet, die Spezifität der IS gegenüber planaren Aufnahmen wesentlich zu steigern [22].

\section{Vorteile der SPECT/CT}

Bereits mit der planaren MPSS und der IS können empfindlich floride Knochenund Protheseninfekte nachgewiesen und aufgrund ihrer Anreicherungscharakteristik von sterilen Entzündungsprozessen unterschieden werden. Schwierigkeiten kann jedoch der Nachweis chronischer Low-grade-Infekte bereiten. Wegen nur geringer Mehrdurchblutung bzw. Leukozytenansammlung in Low-grade-Infekten kann eine sichere Differenzierung von Infekt und steriler Entzündung im Einzelfall nicht möglich sein. Die nuklearmedizinische Infektbildgebung ist daher darauf angewiesen, Teilaspekte mehrerer Modalitäten zu integrieren, um zu einer für klinische Belange ausreichend spezifischen Gesamtaussage zu gelangen. Die SPECT/ CT erfüllt diese Anforderungen und bietet in einem Untersuchungsgang neben der Funktions- und Stoffwechselinformation der Szintigraphie die morphologische Information der Computertomographie. Sie ermöglicht auf diese Weise die Korrelation metabolischer, funktioneller und

Radiologe 2012 · 52:615-620 DOI 10.1007/s00117-011-2272-1

(c) Springer-Verlag 2012

\section{B. Klaeser - M. Spanjol · T. Krause}

\section{SPECT/CT-Infektdiagnostik am Skelett}

\section{Zusammenfassung}

Klinisches/methodisches Problem. Knöcherne Infekte stellen nicht selten eine diagnostische und klinische Herausforderung dar. Radiologische Standardverfahren. Nuklearmedizinische Standardverfahren für die Diagnostik akuter und chronischer Knocheninfekte sind die Mehrphasenskelettszintigraphie und die Infektszintigraphie mit markierten Leukozyten.

Methodische Innovationen. Die Einführung von Hybridtechnologien wie der SPECT/ CT hat die nuklearmedizinische Infektbildgebung tiefgreifend verändert.

Leistungsfähigkeit. Die SPECT/CT er-

laubt bei der Frage nach Knocheninfekten insgesamt eine wesentlich genauere Beurteilung als planare Aufnahmen und SPECT. Bewertung. Die integrierte Akquisition von metabolischer, funktioneller und topographisch-morphologischer Information mit SPECT/CT steigerte insbesondere die Spezifität der Mehrphasenskelettszintigraphie und der Infektszintigraphie mit markierten Leukozyten.

\section{Schlüsselwörter}

Szintigraphie $\cdot$, Single photon emission computed tomography"/CT (SPECT/CT) . Infekt · Osteomyelitis · Prothese

\section{SPECT/CT diagnostics for skeletal infections}

Abstract

Clinical/methodical issue. Skeletal infections are often a diagnostic and clinical challenge.

Standard radiological methods. Nuclear imaging modalities used in the diagnostic workup of acute and chronic skeletal infections include three-phase bone scintigraphy and scintigraphy with labelled leucocytes.

Methodical innovations. The introduction of hybrid technologies, such as single photon emission computed tomography/computed tomography (SPECT/CT) has dramatically changed nuclear medical imaging of infections.
Performance. In general SPECT/CT leads to a considerably more accurate diagnosis than planar or SPECT imaging.

Achievements. Given the integrated acquisition of metabolic, functional and morphological information, SPECT/CT has increased in particular the specificity of three-phase skeletal scanning and scintigraphy with labeled leucocytes.

\section{Keywords}

Scintigraphy · Single photon emission computed tomography/computed tomography · Infection · Osteomyelitis . Prosthesis topographisch-morphologischer Parameter in einem einzigen Untersuchungsgang.

Die SPECT/CT beseitigt die typische methodische Limitation planarer Projektionsaufnahmen, z. B. die räumliche Überlagerung von Befunden, welche die szintigraphische Detektion und Zuordnung kleinerer Anreicherungen unmöglich machen kann [23, 24]. Die CT-Schwächungskorrektur der SPECT-Datensätze verbessert zudem die Erkennbarkeit v. a. kleinerer Befunde in Wirbelsäule und Beckenskelett, deren Anreicherungsintensität in der nichtkorrigierten SPECT unterschätzt wird. Durch die Koregistrierung von SPECT und CT lassen sich Anreicherungen genau lokalisieren, und der Ent- zündungsfokus kann präzise den Weichteilen, Knochenmark, Kortikalis oder Gelenken zugeordnet werden (• Abb. 1a, b). Aufgrund der räumlichen Zuordnung können auch kleinere und geringer anreichernde Befunde nicht nur erkannt, sondern sicher interpretiert werden. Im Vergleich zur planaren Szintigraphie erlaubt die SPECT/CT somit eine spezifischere und detailliertere Beurteilung. Die SPECT/CT erbringt bei $36-53 \%$ der IS einen wesentlichen Beitrag zur endgültigen Diagnose $[5,6]$ und minimiert die Rate an unklaren oder zweifelhaften Untersuchungsresultaten nach planarer und SPECT-Bildgebung $[4,10]$. 

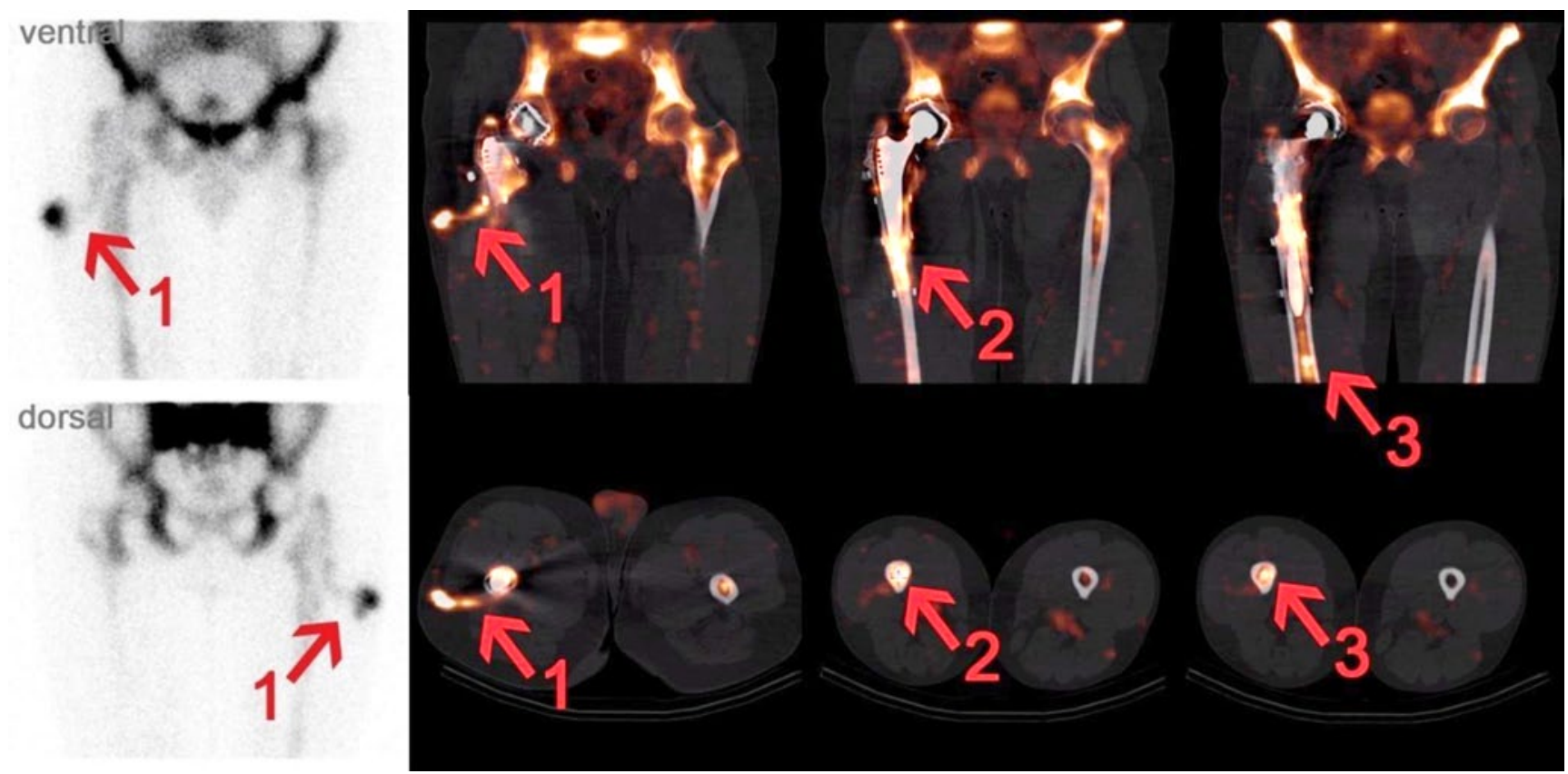

Abb. $3 \Delta$ Rezidivierende Schwellung und Sekretion aus der Narbe nach Hüft-TEP-Implantation rechts vor 7 Jahren. In der planaren IS Darstellung eines Weichteilinfekts mit Fistelgang zur Trochanterregion (1). In der IS-SPECT/CT klare Demarkierung sowohl des Fistelgangs bis zum Femur (1), als auch weiterer moderater Mehranreicherungen im Sinne eines Low-grade-Infekts entlang des Prothesenschafts (2) und distal der Prothese im femoralen Markraum (3). TEP Totalendoprothese, IS Infektszintigraphie, SPECT/CT "single photon emission computed tomography"/CT
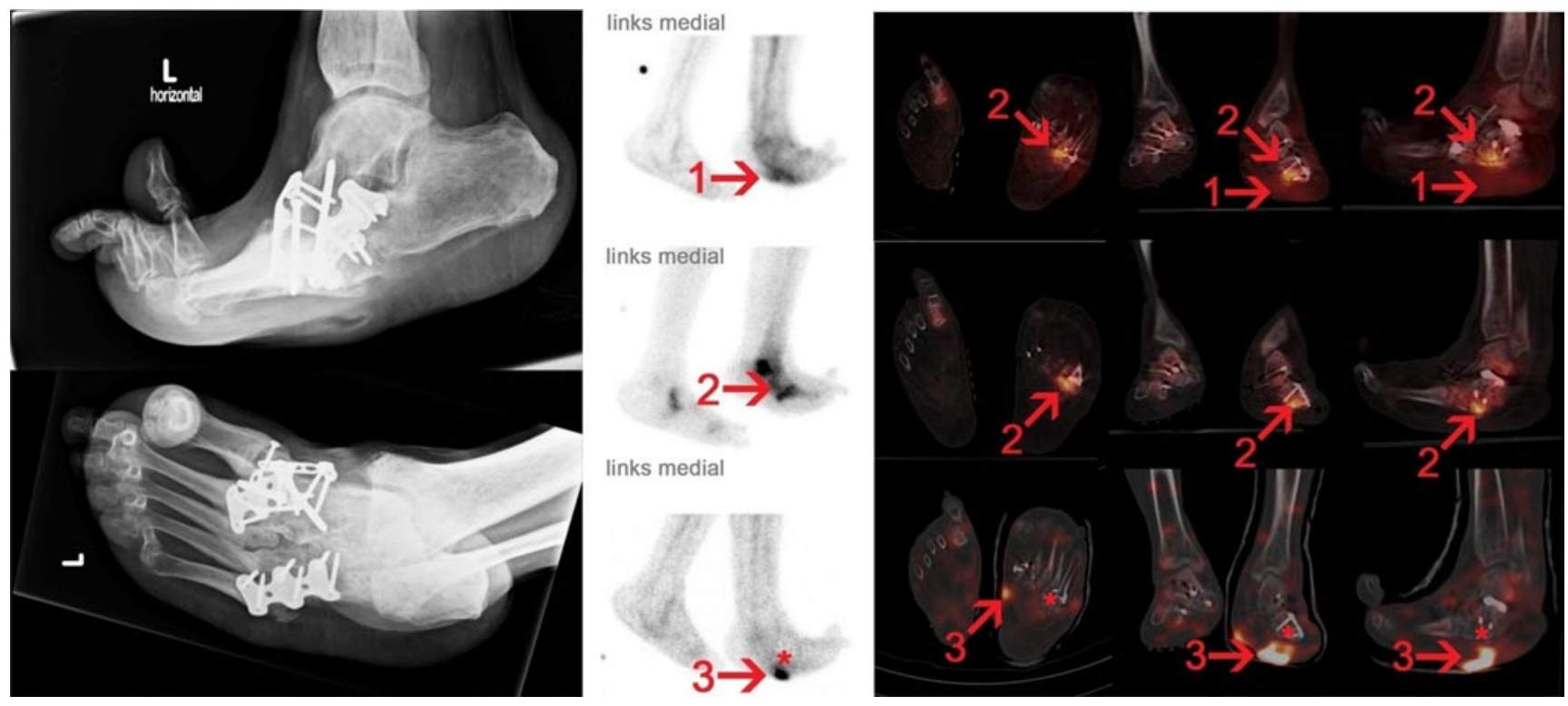

links medial
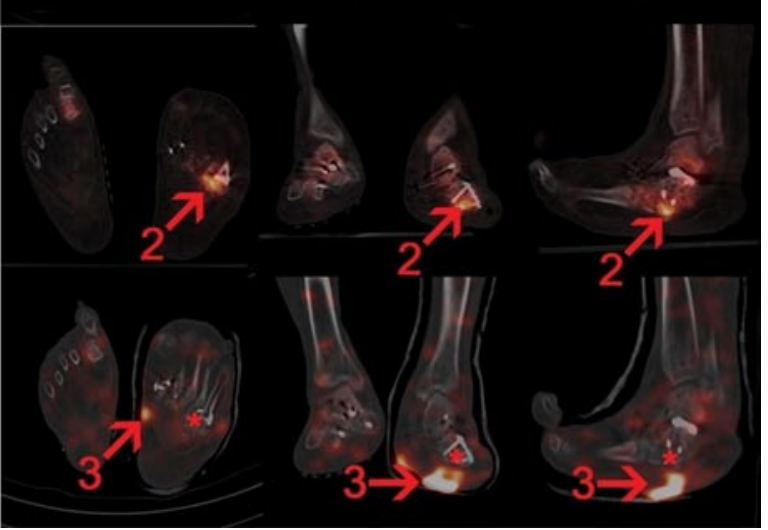

Abb. $4 \Delta$ Zustand nach multiplen osteosynthetisch versorgten Mittelfußfrakturen. Klinisch Verdacht auf Osteomyelitis bei sezernierendem Weichteilinfekt an der Fußsohle. In der MPSS während der venösen Phase (obere Reihe) diffuse Weichteilentzündung der Fußsohle (1). In der MPSS während der ossären Phase (mittlere Reihe) korrespondierend zur venösen Phase gelenkbezogene Mehranreicherung tarsal (2), aktivierte Arthrose oder Infekt. Ansonsten keine suspekten Knochenstoffwechselsteigerungen. In der IS (untere Reihe) Weichteilinfekt der Fußsohle (3). Keine Anhaltspunkte für eine Osteomyelitis, insbesondere nicht gelenkbezogen tarsal $\left(^{*}\right)$. MPSS Mehrphasenskelettszintigraphie, IS Infektszintigraphie

\section{Mehrphasenskelettszintigraphie}

Die Ergänzung der planaren MPSS durch eine SPECT/CT während der ossären Phase erlaubt insgesamt eine genauere
Zuordnung und Beurteilung von Knochenstoffwechselsteigerungen (Spezifität planare Aufnahmen $50 \%$ vs. SPECT/ CT 86\% [9]). Der diagnostische Zugewinn der SPECT/CT beruht sowohl auf der genauen Lokalisation von Knochenstoffwechselsteigerungen als auch auf der Erfassung charakteristischer CT-morphologischer Befunde, bei Osteomyelitis beispielsweise von Osteolysen, periosta- 

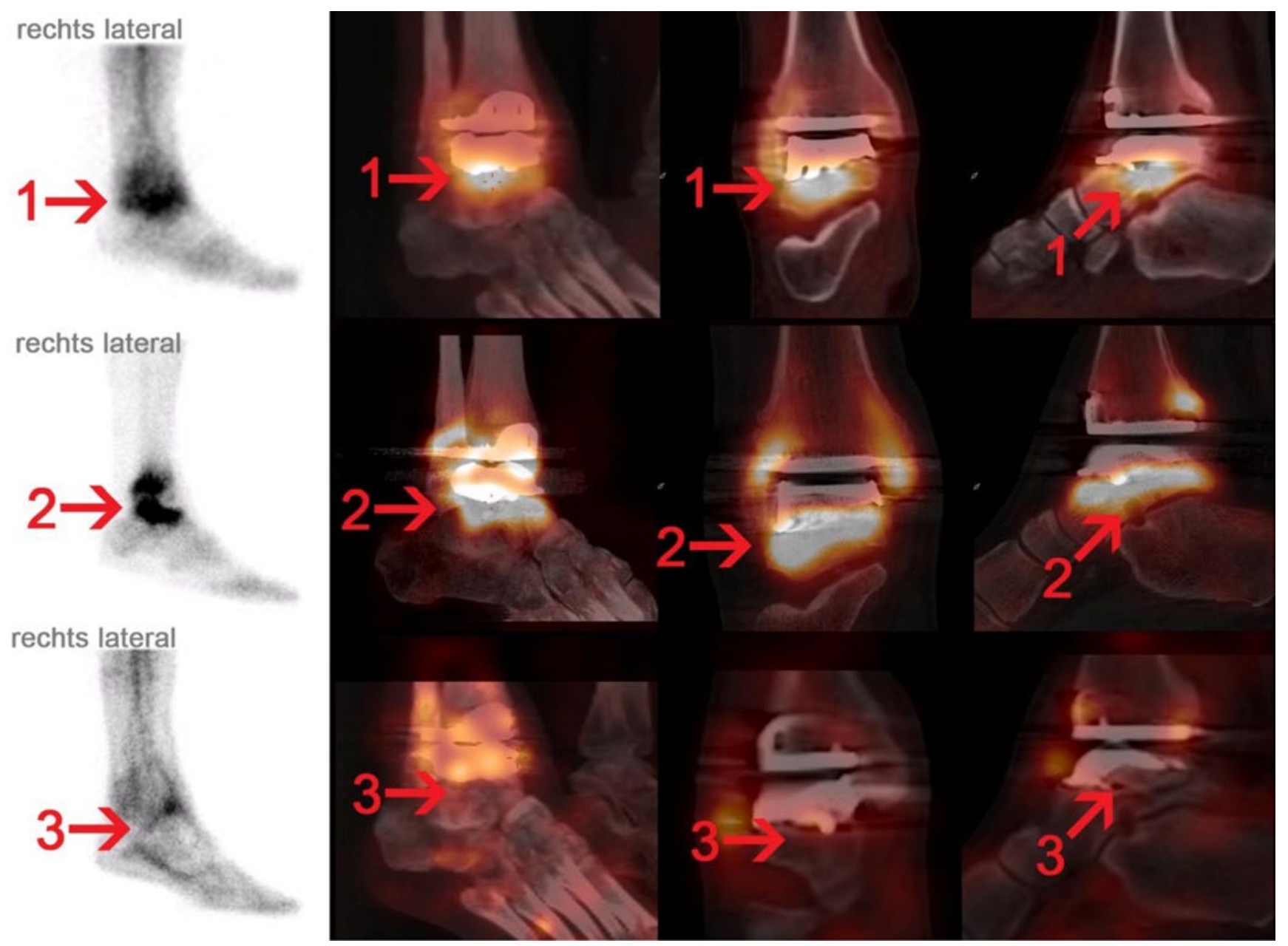

Abb. $5 \Delta$ Zustand nach OSG-TEP-Implantation 2 Jahre zuvor. Zunehmende Schmerzen an der distalen Tibia unter Belastung. In der MPSS während der venösen Phase (obere Reihe) kapselbezogene Anreicherung im Sinne einer Synovialitis sowie intensive Mehranreicherung zentral unter der talaren Prothesenkomponente (1), Protheseninfekt oder reaktive Hyperämie bei Prothesenlockerung. In der MPSS während der ossären Phase (mittlere Reihe) intensive Mehranreicherung unter der gesamten talaren Prothesenkomponente im Sinne einer Lockerung (2) und im Bereich der Malleolengabel, sekundäre ossäre Stressreaktion. In der IS (untere Reihe) Anreicherung kapsulär bei Synovialitis, jedoch in der SPECT/CT keine Anhaltspunkte für einen Protheseninfekt (3): aseptische Lockerung der OSG-TEP. OSG oberes Sprunggelenk, TEP Totalendoprothese, MPSS Mehrphasenskelettszintigraphie, IS Infektszintigraphie, SPECT/CT , single photon emission computed tomography"/CT

len Reaktionen bzw. subperiostalen Knochenformationen oder Sequesterbildungen.

Zusätzliche Vorteile im Rahmen der MPSS bietet eine 2-Phasen-SPECT/CT. Dabei wird während der venösen Phase statt planarer Aufnahmen eine kurze SPECT/CT-Akquisition durchgeführt. Wegen der geringen Detailauflösung der venösen SPECT/CT genügt zur Korrelation und Lokalisation der Mehranreicherungen eine Low-dose-CT-Akquisition mit jeweils technisch niedrigstmöglicher Strahlenexposition. Die SPECT/CTAkquisition während der venösen Phase stellt auch moderate und kleinherdige Steigerungen der Gewebedurchblutung mit höherer Sensitivität dar als planare Aufnahmen. Die MPSS mit 2-PhasenSPECT/CT kann zuverlässig Gelenkkapselanreicherungen bei steriler Synovialitis von Knochen- oder Protheseninfekten abgrenzen. Die genaue Lokalisation venöser Anreicherungen bietet die Grundlage, bei suspekten Befunden eine weiterführende Abklärung mit der IS oder Punktion und Bakteriologie zu lenken (• Abb. 2a, b). Es bleibt in künftigen Studien zu klären, inwieweit die 2-Phasen-SPECT/CT in komplexen Situationen nach Trauma oder Operation auch die Spezifität der MPSS steigern kann.

\section{Infektszintigraphie}

Bei der IS definiert in rund der Hälfte der Fälle erst die SPECT/CT die genaue Ausdehnung des Infekts, unterscheidet sicher zwischen Knochen- und Weichteilinfekten oder ermöglicht die endgültige Diagnose (• Abb. 3; [1]).

Insbesondere nach Trauma, Operation oder bei chronischen Infekten ist die Kombination von MPSS und IS-SPECT/ CT von zusätzlichem Nutzen. Die gemeinsame Beurteilung beider Verfahren bringt eine deutlich höhere diagnostische Genauigkeit (Sensitivität/Spezifität 93/97\% [8]). Einen komplexen Fall, bei dem nach mehreren Frakturen und 
stark veränderter Fussanatomie anhand planarer Aufnahmen ein Knocheninfekt nicht ausgeschlossen werden kann, illustriert $\bullet$ Abb. 4. Hier ist zum einen die topographische Information der IS-SPECT/ CT hilfreich. Zusätzliche Sicherheit bietet jedoch - auf der Basis des hohen NPV der Skelettszintigraphie - die Korrelation mit der MPSS-SPECT/CT während der ossären Phase.

Bei Verdacht auf Protheseninfekt erlaubt die IS-SPECT/CT eine genauere Aussage als die planare IS. Synoviale Anreicherungen im Sinne einer sterilen Synovialitis können sicher gegenüber periprothetischen ossären Anreicherung differenziert und falsch-positive Befunde vermieden werden [5]. SPECT/CT-Aufnahmen ermöglichen ferner den direkten und detaillierten Vergleich der IS mit der MPSSSPECT/CT. Die durch die SPECT/CT gegebene topographische Korrelation macht in der Zusammenschau der Methoden eine sichere Unterscheidung reaktiv-entzündlicher Prozesse bei aseptischer Prothesenlockerung von einer infektbedingten Lockerung möglich ( $\bullet$ Abb. 5).

Die Sensitivität und die Spezifität der IS-SPECT/CT bei Low-grade-Infekten werden mit 89 bzw. 73\%, der NPV mit $94 \%$ angegeben (planare IS 66, 60 und $81 \%$ [7]).

\section{Fazit für die Praxis}

- Die Einführung der SPECT/CT hat die nuklearmedizinische Infektbildgebung tiefgreifend verändert. Die Akquisition metabolischer, funktioneller und topographisch-morphologischer Parameter generiert in einem einzigen Untersuchungsgang eine Fülle von Informationen, die in der überwiegenden Mehrzahl der Fälle eine eindeutige und zuverlässige Diagnose ermöglichen.

- Die MPSS-SPECT/CT besitzt einen sehr hohen NPV und eignet sich daher hervorragend als Eingangsuntersuchung bei Verdacht auf knöcherne Infekte. Die MPSS erfährt durch die 2-PhasenSPECT/CT eine weitere Aufwertung. - Die SPECT/CT im Rahmen der IS mit Antigranulozytenantikörpern steigert durch die exakte topographische Zu- ordnung von Befunden die Spezifität der Untersuchung und erlaubt in der Mehrzahl der Fälle eine Abgrenzung zwischen Infekt- und Knochenmarkanreicherungen.

- Die Kombination von MPSS und ISSPECT/CT erlaubt eine umfassende Gesamtbeurteilung reaktiv-entzündlicher, infektiöser bzw. ossärer Prozesse und verspricht insbesondere in komplexen Situationen eine höhere diagnostische Genauigkeit.

- Die SPECT/CT wird durch metallische Implantate nicht limitiert und ist eine valide Alternative, wenn die MRT in diesem Zusammenhang nicht durchführbar ist; eine abschließende Bewertung der SPECT/CT im Vergleich zu konkurrierenden Methoden wie der PET/CT oder MRT ist nach heutigem Erkenntnisstand noch nicht möglich.

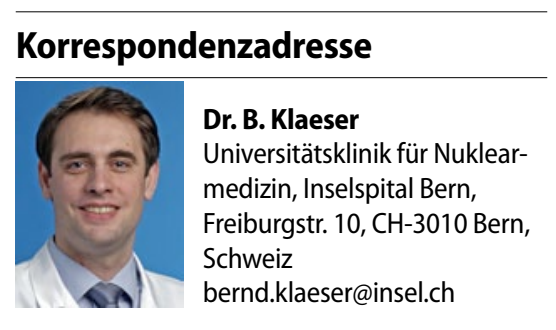

Interessenkonflikt. Der korrespondierende Autor weist auf folgende Beziehung hin: Dr. Klaeser hat Honorare für Vorträge von der Firma Philips AG Healthcare erhalten.

\section{Literatur}

1. Bar-Shalom R, Yefremov N, Guralnik L et al (2006) SPECT/CT using $67 \mathrm{Ga}$ and $111 \mathrm{ln}$-labeled leukocyte scintigraphy for diagnosis of infection. J Nucl Med 47:587-594

2. Becker W, Palestro CJ, Winship J et al (1996) Rapid imaging of infections with a monoclonal antibody fragment (LeukoScan). Clin Orthop Relat Res (329):263-272

3. Capriotti G, Chianelli M, Signore A (2006) Nuclear medicine imaging of diabetic foot infection: results of meta-analysis. Nucl Med Commun 27:757764

4. Djekidel M, Brown RK, Piert M (2011) Benefits of hybrid SPECT/CT for (111)In-oxine- and Tc-99mhexamethylpropylene amine oxime-labeled leukocyte imaging. Clin Nucl Med 36:e50-56

5. Filippi L, Schillaci O (2006) Usefulness of hybrid SPECT/CT in 99mTc-HMPAO-labeled leukocyte scintigraphy for bone and joint infections. J Nucl Med 47:1908-1913
6. Filippi L, Uccioli L, Giurato L et al (2009) Diabetic foot infection: usefulness of SPECT/CT for $99 \mathrm{mTc}$ HMPAO-labeled leukocyte imaging. J Nucl Med 50:1042-1046

7. Graute V, Feist M, Lehner S et al (2010) Detection of low-grade prosthetic joint infections using 99mTc-antigranulocyte SPECT/CT: initial clinical results. Eur J Nucl Med Mol Imaging 37:1751-1759

8. Heiba SI, Kolker D, Mocherla B et al (2010) The optimized evaluation of diabetic foot infection by dual isotope SPECT/CT imaging protocol. J Foot Ankle Surg 49:529-536

9. Horger M, Eschmann SM, Pfannenberg C et al (2007) Added value of SPECT/CT in patients suspected of having bone infection: preliminary results. Arch Orthop Trauma Surg 127:211-221

10. Klaeser B, Spanjol P, Wartenberg J, Krause TM (2008) Bone scan: impact of education and professional experience on the assessment of planar bone scans, SPECT and SPECT-CT. Nuklearmedizin 03:A134

11. Linke R, Kuwert T, Uder M et al (2010) Skeletal SPECT/CT of the peripheral extremities. AJR Am J Roentgenol 194:W329-335

12. Maurer AH, Chen DC, Camargo EE et al (1981) Utility of three-phase skeletal scintigraphy in suspected osteomyelitis: concise communication. J Nucl Med 22:941-949

13. Nelson HT, Taylor A (1980) Bone scanning in the diagnosis of acute osteomyelitis. Eur J Nucl Med 5:267-269

14. Pakos EE, Koumoulis HD, Fotopoulos AD et al (2007) Osteomyelitis: antigranulocyte scintigraphy with $99 \mathrm{mTC}$ radiolabeled monoclonal antibodies for diagnosis - meta-analysis. Radiology 245:732741

15. Palestro CJ, Kipper SL, Weiland FL et al (2002) Osteomyelitis: diagnosis with $(99 \mathrm{~m}) \mathrm{Tc}$-labeled antigranulocyte antibodies compared with diagnosis with (111)In-labeled leukocytes-initial experience. Radiology 223:758-764

16. Prandini N, Lazzeri E, Rossi B et al (2006) Nuclear medicine imaging of bone infections. Nucl Med Commun 27:633-644

17. Romer W, Nomayr A, Uder M et al (2006) SPECTguided $C T$ for evaluating foci of increased bone metabolism classified as indeterminate on SPECT in cancer patients. J Nucl Med 47:1102-1106

18. Rubello D, Rampin L, Banti E et al (2008) Antigranulocyte scintigraphy in infected hip prosthesis: the diagnostic importance of delayed 20-24-h imaging and semiquantitative analysis. Nucl Med Commun 29:994-998

19. Schauwecker DS (1992) The scintigraphic diagnosis of osteomyelitis. AJR Am J Roentgenol 158:918

20. Steinstrasser A, Oberhausen E (1996) Granulocyte labelling kit BW 250/183 - results of the European Multicenter Trial. Nuklearmedizin 35:1-11

21. Turpin S, Lambert R (2001) Role of scintigraphy in musculoskeletal and spinal infections. Radiol Clin North Am 39:169-189

22. Van Acker F, Nuyts J, Maes A et al (2001) FDG-PET, 99mtc-HMPAO white blood cell SPET and bone scintigraphy in the evaluation of painful total knee arthroplasties. Eur J Nucl Med 28:1496-1504

23. Vittorini E, Del Giudice E, Pizzoli A et al (2005) MRI versus scintigraphy with 99mTc-HMPAO-labelled granulocytes in the diagnosis of bone infection. Radiol Med 109:395-403

24. Wagner T, Payoux P, Simon J et al (2010) Discordance between labelled white blood cell scintigraphy and bone scan following suspicion of bone infection: what should be done about it? Nucl Med Rev Cent East Eur 13:5-7 